\title{
A new spin on quantum cryptography: Avoiding trapdoors and embracing public keys
}

\author{
Lawrence M. Ioannou ${ }^{1,2}$ and Michele Mosca ${ }^{1,2,3}$ \\ ${ }^{1}$ Institute for Quantum Computing, University of Waterloo, \\ 200 University Avenue, Waterloo, Ontario, N2L 3G1, Canada \\ ${ }^{2}$ Department of Combinatorics and Optimization, University of Waterloo, \\ 200 University Avenue, Waterloo, Ontario, N2L 3G1, Canada \\ ${ }^{3}$ Perimeter Institute for Theoretical Physics \\ 31 Caroline Street North, Waterloo, Ontario, N2L 2Y5, Canada
}

\begin{abstract}
We give new arguments in support of signed quantum key establishment, where quantum cryptography is used in a public-key infrastructure that provides the required authentication. We also analyze more thoroughly than previous works the benefits that quantum key establishment protocols have over certain classical protocols, motivated in part by the various objections to quantum key establishment that are sometimes raised. Previous knowledge of quantum cryptography on the reader's part is not required for this article, as the definition of "quantum key establishment" that we use is an entirely classical and black-box characterization (one need only trust that protocols satisfying the definition exist).
\end{abstract}

Quantum cryptography 1 has been promoted as a more secure alternative to public-key cryptography based on computational assumptions (see the abstract of Ref. 11 for a typical example). However, an opposing view is sometimes voiced by classical cryptographers and computer security specialists questioning whether quantum cryptography is really a practical way to achieve security against quantum computers, also known as quantum resistance. Several detailed analyses have appeared that consider the benefits and disadvantages of quantum cryptography in comparison to classical alternatives [2, 3, 4, 5]. The present article contributes to the dialogue in a way that we hope is very palatable to the community of quantum-questioning cryptographers: we give new arguments in support of signed quantum key establishment, where quantum cryptography is used in a public-key infrastructure that provides the required authentication.

We also analyze more thoroughly than previous works the benefits that quantum key establishment (QKE) protocols have over certain classical pro-

\footnotetext{
${ }^{1}$ Note that quantum cryptography includes many protocols that this paper does not discuss. We use the term "quantum cryptography" here as a synonym for "quantum key establishment", often called "quantum key distribution" or "QKD".
} 
tocols, motivated in part by the various objections to QKE that have been put forward (for example, in Ref. [5]). Some of those objections follow 2

- Objection 1: Quantum computers are not known to be able to break all classical public-key cryptosystems, such as the McEliece cryptosystem or those based on lattice problems; so we can just upgrade to these quantum-resistant cryptosystems and forget quantum cryptographythat way, we'd retain all the benefits of a public-key infrastructure.

- Objection 2: If all of classical public-key cryptography is found to be easily breakable, then we might as well revert to using our best symmetric-key cryptography, including block ciphers like AES, which we all agree is quantum resistant; quantum cryptography would require symmetric shared initial keys anyway in this case, so it wouldn't gain us anything.

- Objection 3: We don't need any means of key distribution, let alone a quantum mechanical one-let's just exchange a lifetime's worth of symmetric keying material at the start. If for whatever reason we do need new keys, see Objection 4.

- Objection 4: We don't need any means of generating independent secret key over telecommunication links - let's just use a trusted courier each time we need independent secret key.

We address all of these objections.

Not quantum cryptography again. Like in pro-quantum-cryptography articles that have come before this, we assume here that the universe is quantum mechanical, so that, at a minimum, the secret key generated by a secure key-establishment protocol must be secure against an adversary able to perform probabilistic-polynomial-time computations on a quantum computer. As well, as stated by Stebila et al. [4, we "expect the costs and challenges of using [QKE] to decrease to the point where [such] systems can be deployed affordably and their behaviour can be certified." In fact, most of the advantages of quantum cryptography that we point out here have been noted by Paterson et al. 2] or Stebila et al. 44.

Despite these similarities to previous works, our analysis contains distinct new features: it

\footnotetext{
${ }^{2}$ We have stated these objections in our own words.
} 
- suggests a new way to define the classes of classical and QKE protocols, in order to aid their comparison,

- deals properly with the option of using trusted couriers instead of QKE, by distinguishing between in-band and out-of-band actions,

- uses the weakest possible notion of "security" in a quantum universe (i.e. computational security), and therefore does not focus on informationtheoretic security - for its own sake - as an advantage of QKE over computationally-secure classical alternatives,

- provides a finer-grained analysis of the computational assumptions underlying the classical alternatives to QKE,

- highlights a property (we call it "nonattributability") of QKE that has received little attention in the literature, and

- supports a recommendation that is both theoretically and practically sound, which both sides of the "quantum debate" can agree upon.

Generally, we hope the reader finds this article to benefit from a more precise cryptographic analysis, despite its more limited scope in taking an idealized view and thus not discussing the more technological or economical aspects of QKE (including side-channel attacks). In other words, this paper studies the value of the QKE primitive assuming it is available in practice and is as costeffective as any type of "in-band" classical key establishment (see Definition 11) 3 We adopt the same foundational approach that Goldreich does in Refs. [7. 8. This basically means that, when reviewing which computational assumptions are known to be necessary or sufficient for certain cryptographic primitives, we ignore those assumptions (and the schemes based on them) that are ad hoc: we deal only in fundamental computational assumptions, in particular, one-way functions and trapdoor predicates.

But the foregoing analysis is not as complete as it could be. In particular, we do not treat the distributed authenticated key establishment problem

\footnotetext{
${ }^{3}$ The practical availability of the QKE primitive between a typical real-world Alice and Bob is a very non-trivial assumption. For a fairly recent status report on practical QKE systems, one can see Ref. [6], where it is evident that key-rate, distance and availability remain serious obstacles for most practical applications today. In the cases that one believes that QKE could in principle add value, one will need to do an in depth analysis of the various costs and practical limitations before deciding whether in some particular practical situation QKE will be the preferred alternative. Weighing the costs against the value depends on many parameters which vary widely from place to place and over time, and analyzing this broad spectrum is beyond the scope of this paper.
} 
(i.e., in a network setting and where simultaneous, multiple key establishment sessions among many pairs of users are considered) as rigorously as it deserves (e.g. [9, 10]). That is, we implicitly assume that point-to-poin 4 unauthenticated key establishment protocols (whether they be key transport protocols or key agreement protocol:5) and message-authentication protocols (whether they be digital signature schemes or message authentication codes) may be combined in such a way as to form robust distributed authenticated key establishment protocols, without stating the details of how this combining - especially with regard to authenticationactually works 6 This deficiency is manifest in the definition of "security" that we use (Definition 2): it only refers to privacy of the secret key and not its integrity; we take authentication in a network-setting for granted (for both classical and quantum networks). Thus, analyzing point-to-point key establishment systems is sufficient for our scope and, for such systems, integrity of the established secret key is obtained either by assumption (in the case of unauthenticated key establishment) or by the message-authentication protocols used to authenticate the classical communication channel (in the case of authenticated key establishment). Our omission of the analysis of distributed QKE in no way is meant to imply that the problem is trivial - we believe it is an important open problem, which to our knowledge has not been addressed in any previous works.

As a final note to the reader, we stress that previous knowledge of quantum cryptography is not required for this article. The definition of "QKE" that we use is an entirely classical and black-box characterization (one need only trust that protocols satisfying the definition exist).

Key establishment. We are ultimately interested in authenticated key establishment (or AKE), since, in practice, it is usually not a reasonable

\footnotetext{
${ }^{4}$ By "point-to-point" protocols or key establishment systems we mean those that presume a unique pair of honest participants in the protocol; in other words, Alice and Bob are fixed.

${ }^{5}$ Recall that a key transport protocol is a key establishment protocol where the final secret key is generated by one party and sent to the other party (using some kind of encryption mechanism). By contrast, a key agreement protocol is a key establishment protocol where both parties contribute to the generation of the final secret key. See Ref. [11] for more details.

${ }^{6}$ We follow Ref. 11 in our use of the terms "authenticated (key establishment)" and "unauthenticated (key establishment)". In this convention, the word "(un)authenticated" describes the guaranteed condition of the final shared key resulting from the protocol. We note that this convention is the opposite of that in Ref. 8], where "(un)authenticated" describes the a priori assumption on the (classical) communication channel used in the protocol.
} 
assumption that the classical channel connecting Alice and Bob is authenticated a priori. But we shall also consider unauthenticated key establishment (or UKE), because, as well as being useful as a building block for AKE systems, it is an often-considered cryptographic primitive in more foundational works, e.g., Ref. [12] (see Remark 2). We now make some precise definitions.

A (point-to-point) AKE system consists of two probabilistic-polynomialtime (quantum) computers, called "Alice" and "Bob", that

- are preloaded with classical initial keys, $k_{A}$ (stored on Alice) and $k_{B}$ (stored on Bob), which are pre-distributed out of band (see Definition 11) in an authenticated and, where necessary (for example, when the keys are symmetric), private fashion, and

- are connected by two insecure channels, one quantum and one classical, variously monitored or controlled by an adversarial probabilisticpolynomial-time (quantum) computer, called "Eve", and

- together execute a particular (point-to-point) AKE protocol, the specification $\pi$ of which is preloaded authentically but is not secret, and

- which results in Alice and Bob computing outputs $s_{A}$ and $s_{B}$, respectively, such that either $s_{A}=s_{B}=\perp$, which corresponds to Alice and Bob aborting the protocol, or $s_{A}$ and $s_{B}$ are bit-strings, in which case, if $s_{A}=s_{B}$, then the secret key $s:=s_{A}$ is defined.

When the initial keys are symmetric $\left(k_{A}=k_{B}\right)$, we may use $k$ to denote each one, i.e., $k=k_{A}=k_{B}$; if the initial keys are asymmetric $\left(k_{A} \neq k_{B}\right)$, then

$$
\begin{aligned}
k_{A} & =\left(x_{A}, y_{B}\right) \\
k_{B} & =\left(x_{B}, y_{A}\right),
\end{aligned}
$$

where $\left(x_{A}, y_{A}\right)$ is Alice's private-public key-pair and $\left(x_{B}, y_{B}\right)$ is Bob's privatepublic key-pair. We will say more about asymmetric (public-key) cryptography later on.

Definition 1 (In band/out of band). The term "in band" describes actions carried out in the normal course of telecommunications strictly via remote signalling across communication channels. The term "out of band" is used to mean "not in band" and describes communication via non-digital/manual means as opposed to via standard telecommunication devices. 
Remark 1 (Classical channel). Strictly speaking, there is no need for a dedicated classical channel between Alice and Bob, since classical information can be sent along the quantum channel. However, the well-known QKE protocols (i.e., those based on the ones in Refs [13, 14]) clearly distinguish the classical from the quantum communication; in particular, it suffices that only the classical communication is authenticated in order for the secret key to be authenticated at the end of the protocol (whereas, one could imagine a quantum protocol where the quantum communication also needs to be authenticated). In line with this distinction, we assume separate quantum and classical channels.

A (point-to-point) UKE system is defined similarly to an AKE system, with only the following differences:

- Alice and Bob possess no initial keys and

- the classical channel is assumed to be authenticated, i.e., Eve is assumed only to passively monitor the classical channel (but she can still totally control the quantum channel), and

- $\pi$ is a (point-to-point) UKE protocol.

We also need to define conditions under which a key establishment protocol is secure or, more specifically, quantum-resistant. We would like a definition that applies equally well to both quantum and fully classical protocols, i.e., all protocols allowed in the above frameworks. Since we take authentication for granted (as explained above), the following security definition is sufficient for both AKE and UKE systems. Call a key establishment protocol perfectly secure if, for any algorithm for Eve, we have that (1) $s_{A}=s_{B}$, (2) if $s_{A} \neq \perp$ then $s_{A}$ is uniformly distributed and independent of Eve's state, and (3) if Eve does not interfere with the protocol (where we assume otherwise perfect channels), then $s_{A} \neq \perp$. Let $\mathcal{I}$ be an ideal key establishment system that implements a perfectly secure protocol. Let $\mathcal{R}(\pi)$ be a real key establishment system that uses protocol $\pi$. Let $n$ be the minimum length of the secret key $s$ if Alice and Bob do not abort. Consider a probabilisticpolynomial-time (quantum) distinguisher running in time polynomial in $n$, that interacts with either $\mathcal{I}$ or $\mathcal{R}(\pi)$ and then outputs a guess bit $B$; the distinguisher has access to Eve's system and the outputs $s_{A}$ and $s_{B}$.

Definition 2 (Quantum-resistant key-establishment protocol (with respect to privacy)). Assuming the above definitions, a point-to-point key-establishment 
protocol $\pi$ is quantum-resistant (with respect to privacy) if, for any such distinguisher, the quantity

$$
|\operatorname{Pr}[B=1 \mid \mathcal{I}]-\operatorname{Pr}[B=1 \mid \mathcal{R}(\pi)]|
$$

is negligible for all sufficiently large $n$, where $\operatorname{Pr}[B=1 \mid \mathcal{I}]$ and $\operatorname{Pr}[B=$ $1 \mid \mathcal{R}(\pi)]$ are the probabilities that $B=1$ when the distinguisher interacts with $\mathcal{I}$ and $\mathcal{R}(\pi)$, respectively.

We give this (semi-formal) definition for completeness; we refer the reader to Refs [15, 16, 7, 17] for how to rigorize such a definition.

As a final specification of our basic setup, it will be helpful to define the classical communication $c$ in a key establishment protocol. For classical protocols, the classical communication is all the communication between Alice and Bob. For arbitrary (quantum) protocols, defining the classical communication is a bit more subtle; we refrain from giving a formal definition here (for the sake of the reader who may be unfamiliar with quantum measurement). Rather, for the quantum protocols we care about, it suffices to define the classical communication tautologically as the classical communication specified in the protocol, since these protocols clearly and naturally distinguish the classical and quantum information sent between Alice and Bob.

The contenders. Below are listed and defined two main classes of pointto-point UKE protocols as well as the five main classes of point-to-point AKE protocols that are considered in the literature when evaluating the usefulness of quantum cryptography in comparison to classical techniques for key establishment. These classes, as defined, do not cover all conceivable protocols, but do cover all the ones that are usually considered (which suffices here). In defining these classes, we restrict to quantum-resistant protocols (because the universe is quantum). It will help to view the quantities $k_{A}$, $k_{B}, k, s$, and $c$ introduced above as random variables. For example, in the case of symmetric initial keys, the quantity $k$ may be viewed as a uniformly distributed random variable in $\{0,1\}^{\ell}$, for some fixed $\ell \in \mathbb{Z}^{>0}$ that determines the length of the initial keys.

Unauthenticated key establishment protocols:

- Classical UKE (c-UKE) - This class includes any quantum-resistant and totally classical UKE protocol. It includes unauthenticated key transport protocols based on public-key encryption (but not those based on symmetric-key encryption). 
- Quantum UKE (q-UKE) - This class includes any quantum-resistant UKE protocol such that, whenever Eve has not interfered with the protocol, the secret key $s$ is independent of the classical communication $c$, i.e., for all values $c^{\prime}$ of the classical communication and all values $s^{\prime}$ of the secret key,

$$
\operatorname{Pr}\left[s=s^{\prime} \mid c=c^{\prime}\right]=\operatorname{Pr}\left[s=s^{\prime}\right] .
$$

It includes the well-known QKE protocols and can easily be shown not to include any classical protocols 7

Remark 2 (Secret key agreement). The cryptographic primitive realized by protocols in c-UKE is usually referred to as secret key agreement (or sometimes just secret agreement) in the literature. Note that this primitive is also realized by protocols in q-UKE.

Authenticated key establishment protocols:

- Out-of-band key establishment (OOB) - This class includes any AKE protocol where Alice and Bob are preloaded with the secret key out of band, i.e.,

$$
s=k_{A}=k_{B} .
$$

It includes protocols that employ a trusted courier. The initial keys in such protocols are typically much larger than in protocols belonging to the classes below.

- Pseudorandom generator expansion (PGE) - This class includes any quantum-resistant and totally classical AKE protocol not in OOB that uses symmetric initial keys where Alice and Bob establish a secret key that is efficiently computable from the initial keys, i.e., there exists a deterministic-polynomial-time classical algorithm $A$ such that

$$
s=A(\pi, k) .
$$

\footnotetext{
${ }^{7}$ We sketch a proof of the latter fact that no purely classical protocol can be quantum resistant and satisfy (4). Let $r_{A}$ and $r_{B}$ be binary strings encoding the private local randomness that Alice and Bob respectively use in the protocol. Consider the sequence $c_{1}, c_{2}, \ldots$ of messages passed between Alice and Bob. Each $c_{i}$ places constraints on the values of $r_{A}$ and $r_{B}$. Since, at the end of the protocol, the secret key $s$ is uniquely determined, it must be that $r_{A}$ and $r_{B}$ are determined by the classical communication $c$ up to implying a unique $s$, i.e., $H(s \mid c)=0$, where $H$ is the Shannon entropy. For any two random variables $X$ and $Y, H(X \mid Y)=H(X)$ if and only if $X$ and $Y$ are independent 18. Therefore, if (4) holds, then $H(s)=H(s \mid c)=0$, so that $s$ is a constant and thus the protocol is not quantum resistant.
} 
It includes protocols that use a pseudorandom generator to expand the initial keys into a secret key.

- Weak classical AKE (wc-AKE) - This class includes any quantum-resistant and totally classical AKE protocol in neither PGE nor OOB that uses symmetric initial keys. Note such protocols have the property that the secret key is efficiently computable from the initial keys and the communication, i.e., there exists a deterministic-polynomial-time classical algorithm $A$ such that

$$
s=A(\pi, k, c) .
$$

The class includes authenticated key transport protocols based on symmetric-key encryption.

- Strong 8 classical AKE (sc-AKE) - This class includes any quantumresistant and totally classical AKE protocol, where Alice and Bob establish an authenticated secret key $s$ that is not functionally dependent on the initial keys $k_{A}$ and $k_{B}$, i.e., there exists a deterministic-polynomialtime classical algorithm $A$ such that

$$
s=A\left(\pi, r_{A}, r_{B}\right),
$$

where $r_{A}$ and $r_{B}$ are (random variables representing) the private local random choices of Alice and Bob respectively (made independently of the initial keys). It includes authenticated key transport protocols based on public-key encryption (but not those based on symmetric-key encryption); more generally, it includes the "authenticated version" of any quantum-resistant UKE protocol, where the initial keys are used (only) to authenticate all the communication of the protocol (see Remark (7).

- Quantum AKE (q-AKE) - This class includes any quantum-resistant AKE protocol such that, whenever Eve has not interfered with the protocol, the secret key $s$ is independent of the initial keys and the classical communication $c$, i.e., for all values $k_{A}^{\prime}$ and $k_{B}^{\prime}$ of the initial

\footnotetext{
${ }^{8}$ Our use of the word "strong" differs from that in Ref. 19, where a key establishment protocol is secure only if it remains secure under the reveal of any subset of the initial (also called "long-term") and ephemeral keys that does not contain both the initial and ephemeral keys of one of the parties. The protocols of the class we define here need only remain secure under the reveal of the initial keys. Indeed, the "strong" of Ref. [19] is stronger than ours.
} 
keys and all values $c^{\prime}$ of the classical communication and all values $s^{\prime}$ of the secret key,

$$
\operatorname{Pr}\left[s=s^{\prime} \mid k_{A}=k_{A}^{\prime}, k_{B}=k_{B}^{\prime}, c=c^{\prime}\right]=\operatorname{Pr}\left[s=s^{\prime}\right] .
$$

It includes the authenticated version of the well-known QKE protocols and can easily be shown not to include any classical protocols (similarly to the class q-UKE, defined previously).

Remark 3 (Possible emptiness of classical classes). Of the classes of inband key establishment protocols, only q-UKE and q-AKE are known to be nonempty.

Remark 4 (Key pre-distribution v. dynamic key establishment). The union of the classes $\mathrm{OOB}$ and PGE contains protocols referred to collectively as key pre-distribution schemes [11], which is why we label these two classes differently. Note that there is no need to authenticate the in-band communication in these protocols because there is none. Protocols that are not key pre-distribution schemes are said to accomplish dynamic key establishment.

Remark 5 (Definition of sc-AKE). The class sc-AKE may contain protocols that use the "quantum public-key cryptosystems" in Ref. [20], since the model does not stipulate how initial keys are derived (i.e., they could be derived using a quantum computer).

Remark 6 (Definition of q-AKE). The class q-AKE may contain protocols obeying physical theories other than quantum theory.

Remark 7 (UKE implies AKE). Note that if $\pi$ is in C-UKE, then $\pi$ naturally gives rise to a protocol in Sc-AKE when combined with a secure classical message-authentication protocol. A similar statement holds for q-UKE and q-AKE.

We subdivide the classes sc-AKE and q-AKE by the type of initial keys - either symmetric or public - used in the particular key establishment protocol, i.e., we have the following disjoint unions

$$
\begin{aligned}
\mathrm{sc}-A K E & =\mathrm{sc}-A K E_{\text {sym }} \cup \mathrm{sc}-A K E_{\text {pub }} \\
\mathrm{q}-A K E & =\mathrm{q}-A K E_{\text {sym }} \cup \mathrm{q}-A K E_{\text {pub }} .
\end{aligned}
$$

Table 1 summarizes the different classes by the various categories. 


\begin{tabular}{|c||c|c||c|}
\hline & UKE & AKE & \\
\hline \hline \multirow{2}{*}{ key pre-distribution } & - & OOB & out-of-band \\
\cline { 2 - 4 } & - & PGE & in-band \\
\hline dynamic & - & wc-AKE & \\
key establishment & c-UKE & sc-AKE & \\
& q-UKE & q-AKE & \\
\hline
\end{tabular}

Table 1: The different classes of key establishment protocols.

Apples and Oranges. The class OOB is included in the above list (and in the following analysis) largely for completeness; it is not technically considered a key establishment protocol. Out-of-band protocols for key establishment need not employ any fundamental cryptographic primitives and cannot provide the same essential functionality that in-band protocols do, i.e., generating new secret key in band. The generally accepted view is that out-of-band key establishment is the most secure way to establish potentially very long secret keys, but that well-implemented in-band protocols typically provide either a more feasible solution in particular applications or a more cost-effective solution in the long term. Because we are making the (reasonable) assumption that QKE will be cost-effective in the future, it reasonably follows that, in at least some cases, it will also be more cost-effective than out-of-band key establishment in the future. We mean to challenge here previous comments made by Bernstein [5], that trusted couriers perform equally as well as QKE systems insofar as their ability to generate entropy in the cryptographic system (from Eve's point of view). The distinction between in-band and out-of-band entropy generation is an important one (cost-wise), and it is impossible to generate entropy in band using classical cryptography alone.

Computational assumptions. We would like to closely examine the fundamental computational assumptions that underlie the various kinds of key establishment protocols. To do this, we start by recalling the following wellknown theorems 9

\footnotetext{
${ }^{9}$ The following theorems and other similar statements should be interpreted as follows. A statement of the form "Cryptographic objects of type $Y$ exist if cryptographic objects of type $X$ exist" means "If there exists an object of type $X$, then there exists an object of type $Y$ such that breaking the object of type $Y$ implies breaking the object of type $X$." Such a statement may also be phrased, " $X$ implies $Y$ ".
} 
Theorem 8 ([7]). Pseudorandom generators exist if and only if one-way functions exist.

Theorem 9 ([8]). Symmetric-key encryption schemes exist if and only if one-way functions exist.

Theorem 10 ([21]). Public-key encryption schemes exist if and only if trapdoor predicates exist.

Theorem 11 ([22]). Information-theoretically-secure symmetric-key message authentication codes exist.

Theorem 12 ([23, 24]). Public-key signature schemes exist if and only if one-way functions exist.

Theorem 13 (25]). Information-theoretically-secure q-UKE-protocols exist.

Because we are assuming a quantum universe, one-way functions and trapdoor predicate 10 in this article (if they exist) are secure against an adversary with a quantum computer, but are still assumed to be efficiently computable on a classical computer; also, trapdoors are still considered to be classical objects 11 We also note that Theorems 8, 9, 10, and 12 hold with respect to black-box reductions: if the theorem states that $X$ implies $Y$, then $Y$ can be constructed from $X$, only using $X$ as a black box, i.e., the reduction does not rely on the specifics of how $X$ works; furthermore, the security reduction is also a black-box one, i.e., an algorithm for breaking $X$ can be constructed from a black box for breaking $Y$. Non-black-box theorems of this sort are also possible (for example, see Ref. [27]), but are rarely required for these kinds of results, and indeed are not required for the theorems we quote. This is lucky, since it guarantees us that the theorems still hold with respect to a quantum universe.

The theorems establish the minimal fundamental computational assumptions known to be sufficient for the existence of protocols by class, summarized in Table 2. Public-key encryption implies one-way functions [8].

\footnotetext{
${ }^{10}$ Informally, the predicate $B(x) \in\{0,1\}$ is a(n) (unapproximable) trapdoor predicate if anyone can find an $x$ such that $B(x)=0$ or a $y$ such that $B(y)=1$ efficiently on a classical computer, but only one who knows the trapdoor can, given $z$, compute $B(z)$ efficiently on a quantum computer (this notion was introduced in Ref. 21]). Note that one can use a trapdoor predicate for public-key encryption: the bit $b$ is encrypted as any $x$ such that $B(x)=b$.

${ }^{11}$ One could consider "one-way/trapdoor quantum functions", where the input and output of the functions are classical or quantum, and the functions only need to be computable efficiently on a quantum computer. We stick to classical one-way functions and trapdoor predicates that are quantum resistant, candidates of which are, e.g., the trapdoor predicates underlying some lattice-based cryptosystems (see Ref. 26] for more examples).
} 


\begin{tabular}{|c||c|}
\hline Protocol class & Computational assumptions \\
\hline \hline OOB & none \\
PGE & one-way functions \\
wc-AKE & one-way functions \\
c-UKE/sc-AKE & trapdoor predicates \\
q-UKE/q-AKE & none \\
q-AKE & one-way functions \\
\hline
\end{tabular}

Table 2: Minimal known fundamental computational assumptions sufficient for the existence of key establishment protocols in each class.

Thus, the classes c-UKE and sc-AKE require the strongest assumption in the table - the existence of trapdoor predicates - which reflects the fact that it is not known how to construct any protocol in these classes without relying on (or implying) public-key encryption 12 To facilitate our discussion, we summarize this point as the following conjecture:

Conjecture 14 (Classical secret key agreement implies public-key encryption). Every protocol in c-UKE implies a trapdoor predicate (with respect to a possibly-non-black-box reduction).

Safest fair comparison. Most articles on quantum cryptography that appeared in the 1990s and early 2000s stressed the fact that q-AKE sym (respectively, q-UKE) is the only known class of in-band AKE (respectively, UKE) protocols that requires no computational assumptions. But implicitly discarding all computational assumptions in this way makes it impossible to have a serious discussion about the relative merits of classical and quantum protocols for key establishment (since any classical key-establishment protocol requires some computational assumption). So, suppose we give classical cryptography a fighting chance: suppose we allow only the weakest computational assumption necessary for in-band classical key establishment-oneway functions.

There is good reason to do this. Trapdoor predicates seem to be inherently less secure than one-way functions in general. Firstly, trapdoor

\footnotetext{
${ }^{12}$ One might declare Table 2 misleading, since, for example, Theorem 12 is usually regarded merely as a plausibility result: the construction of a signature scheme from an arbitrary one-way function is relatively very inefficient. To address this issue, we note that reasonably practical constructions are known for pseudorandom generators, symmetric-key encryption schemes, and signature schemes from one-way permutations [7, 8]. Thus, even restricting to reasonably practical schemes, the class sc-AKE still requires the assumption of a primitive possessing a trapdoor property, as far as we know.
} 
predicates easily imply one-way functions [8], whereas the converse is believed not to be true. As some evidence for this, we note that it has been shown in Ref. [12] that, with respect to black box reductions (and with respect to a classical universe), one-way functions are not sufficient (even) to imply secret key agreement (see Remark 2, but we have not checked that this theorem holds with respect to a quantum universe - in general, such classical black-box no-go theorems need not). Secondly, using the equivalences stated in Theorem 9 and Theorem 10, it seems far more likely that an efficient algorithm would be found for breaking a public-key cryptosystem (i.e. computing a trapdoor predicate) than breaking a symmetric-key cryptosystem (i.e. inverting a one-way function without the trapdoor property), because the public-key cryptosystem possesses more structure in order to embed a trapdoor into the encryption "function". Quantum computers are firmly believed not to be able to invert all one-way functions efficiently; we state this as a conjecture:

Conjecture 15 (One-way functions exist). Quantum-resistant one-way functions (computable in polynomial-time on a classical computer) exist.

We do not mean to suggest that quantum-resistant trapdoor predicates do not exist (we don't know). We do suggest, though, that the added structure of trapdoor predicates makes it much more likely that algorithms for the underlying problems will improve at a more unpredictable rate: plain oneway functions are less risky.

Even allowing one-way functions, we see that QKE has advantages over classical systems, beyond unconditional security.

Advantages of QKE assuming (only) one-way functions. Most of the advantages below have appeared elsewhere in the literature in one form or another, but our presentation is motivated differently. The following four advantages are not intended to be totally independent; indeed, each is just a qualitatively different consequence of the fact that the secret key is independent of both the initial keys and classical communication in QKE (and that we have taken sc-AKE-protocols out of the picture).

- Advantage 1: Improved security against reveal of initial keys

In classical cryptography, the physical nature of a cryptosystem and protocol leads to the consideration of different types of attacks, some more serious or more technologically difficult to mount than others. Similarly, 
adversaries are often categorized by their power, for example, passive adversaries are considered only to be able to read certain data that is sent along a channel, whereas active adversaries are assumed to have complete control over the channel. It is also relevant to consider precisely when Eve may become active; a delayed adversary is one that remains passive until the key establishment protocol completes, but is active immediately afterwards.

The physical nature of a QKE system leads to the consideration of new kinds of attacks and adversaries. Because of the two different channels used, Eve can now operate differently on these two channels 13 Thus an adversary can be defined by whether it is passive, delayed, or active on the classical and quantum channels respectively; e.g., (p,p) means "passive on both channels" and $(\mathrm{a}, \mathrm{d})$ means "active on the classical channel and delayed on the quantum channel".

With these terms in place, Table 3 shows how q-AKE-protocols have advantages over the other classical protocols that also assume (at most) oneway functions, for certain types of adversary; the table indicates whether secure key can be established when the initial keys have been revealed. For any situation where an immediate active attack is not deployed for whatever reason (e.g. not technologically feasible, or not a high priority at the time), a passive adversary who knows the initial keys loses the ability to compromise the secret key later should she become an active attacker later. Note that if "sc-AKE" appeared in the leftmost column of the table, the corresponding row of "yes" / "no" values would look the same as the row corresponding to the class q-AKE.

Note that, in order to break a q-AKE-protocol - or, more precisely, break the cryptosystem that comprises the q-AKE-protocol-Eve, knowing all the initial keys, can mount an active and sustained "man-in-the-middle" attack; furthermore, for a $\mathrm{q}-\mathrm{AKE}_{\mathrm{sym}}$-system, the active attack must occur during the first instance of the protocol (as any subsequent instance will use different and independent initial keys). In large networks, this may pose a considerable challenge for Eve, depending on when she learns the initial keys and whether the connections among users are fixed or ad-hoc.

Remark 16 (Perfect forward secrecy). Note that Advantage 1 is different from perfect forward secrecy, a much weaker notion referring to whether secret keys established in past sessions (with old initial keys no longer stored on Alice and Bob) are secure once current initial keys are revealed. While q-

\footnotetext{
${ }^{13}$ We define "passive" on the quantum channel to mean having no access, since it is difficult to formulate a definition of "read only" for a quantum channel. Measurement, which seems necessary for reading, is an active process.
} 


\begin{tabular}{|c||c|c|c|c|c|}
\hline & $(\mathrm{p}, \mathrm{p})$ & $(\mathrm{d}, \mathrm{d})$ & $(\mathrm{a}, \mathrm{p})$ & $(\mathrm{a}, \mathrm{d})$ & $(\mathrm{a}, \mathrm{a})$ \\
\hline OOB & no & no & no & no & no \\
PGE & no & no & no & no & no \\
wc-AKE & no & no & no & no & no \\
q-AKE & yes & yes & yes & yes & no \\
\hline
\end{tabular}

Table 3: Security against reveal of initial keys. The entries (yes/no) of the chart indicate whether the secret key generated from the key establishment protocol is secure under the reveal of either Alice's or Bob's initial key for the given adversary (see the main text for an explanation of the notation used to define the adversaries). The class sc-AKE does not appear, since we are not assuming trapdoor predicates (and there is no known sc-AKE-scheme that does not imply trapdoor predicates).

AKE-protocols certainly have perfect forward secrecy, Bernstein [5] has noted that well-implemented PGE-protocols do, too.

- Advantage 2: Reduced dependence on out-of-band actions

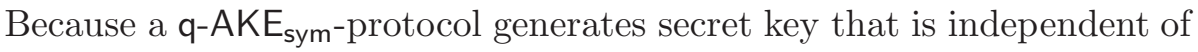
the initial keys and the classical communication, initial keys can be smaller in the $\mathrm{q}-\mathrm{AKE}_{\mathrm{sym}}$-protocol than in an OOB-protocol, i.e., less initial entropy is needed to prime the system. Also, a q-AKE $\mathrm{E}_{\text {sym-system may require fewer }}$ subsequent out-of-band actions for refreshing initial keys, compared to PGEand wc-AKE-systems (at the very least because the latter are more vulnerable to initial-key-reveal attacks - see above).

- Advantage 3: Reduced dependence on trusted third parties

In a network, key establishment can be done in a mediated fashion, via a trusted key distribution centre, whose job is to give session keys to Alice and Bob so that they may communicate securely. As part of the setup, every user in the network, including Alice and Bob, shares an initial key (established out of band) with the key distribution centre; in principle, these initial keys may be asymmetric or symmetric. An example of such a system is Kerberos, where the initial keys are symmetric, and, upon request by either Alice or Bob, the key distribution centre generates a symmetric key and sends it (encrypted using the initial keys) to Alice and Bob, who then use it to encrypt and decrypt messages between each other.

Quantum key establishment may also be done in a mediated fashion, so that the channels connecting Alice to Bob go through a key distribution 
centre, which gives Alice and Bob a session key to be used as a symmetric initial key in a q-AKE $\mathrm{E}_{\text {sym }}$-protocol.

If trapdoor predicates are not assumed to exist, then any classical mediated key establishment system must use symmetric initial keys; this is because the key distribution centre must send keys to Alice and Bob, and these keys must be, at least partially, encrypted (assuming the key distribution centre is not to play an active part in the communication between Alice and Bob). Similarly, the session keys must be symmetric keys, too.

Comparing any classical mediated key establishment system to one where Alice and Bob use their symmetric session keys as initial keys in a q-AKE $\mathrm{E}_{\text {sym }}{ }^{-}$ protocol, we see that, in the quantum case, Alice and Bob do not need to trust the key distribution centre after their key establishment protocol is complete. By contrast, in the classical case, the key distribution centre must always be trusted, since it knows the keys that Alice and Bob use to communicate securely. As well, Alice and Bob may be able to decouple themselves completely from the key distribution centre after their first q$A K E_{\text {sym-session. Thus, any compromise of the key distribution centre after }}$ the first q-AKE $\mathrm{E}_{\text {sym }}$-session does not necessarily affect Alice and Bob.

- Advantage 4: Long-term security from short-term security

The secret key generated by any q-AKE-protocol will be informationtheoretically secure even if the authentication algorithm is broken in the short term - as long as the break occurs after the key establishment protocol is completed. We may refer to this as "conditional information-theoretic security". This allows for the use of authentication algorithms that are perhaps less secure in the long term but are easier to manage with regard to initial keys, i.e., public-key algorithms. Note that any $q-A K E_{\text {pub-system has }}$ the extra advantage over a $\mathrm{q}-\mathrm{AKE}_{\mathrm{sym}}$-system that it is less susceptible to running out of authentication key due to noise or eavesdropping, because there is no practical limit on how many classical messages may be authenticated. In other words, using public-key authentication guards against at least one type of denial-of-service attack.

Also, Alice and Bob may not need to rely on the same type of authentication used for the first q-AKE-session for subsequent q-AKE-sessions, i.e., for the first session, Alice and Bob may execute a $q-A K E_{\text {pub-protocol, but, for }}$ all subsequent sessions (in principle, i.e., in the absence of sufficiently heavy adversarial action or noise), they may execute a $\mathrm{q}-\mathrm{AKE}_{\text {sym-protocol. Two }}$ potential advantages of such a two-phase system are that (1) subsequent key establishment sessions may run faster (since the symmetric-key algorithms 
may be more efficient than public-key algorithms for the required level of security) and (2) subsequent key establishment sessions may not need to rely on any computational assumptions.

If quantum computers can be assumed not to exist in the short term, i.e., for the service-lifetime of the public keys, then one can even use public-key signature schemes whose security relies on the assumption of hardness of factoring and the discrete logarithm problem for classical computers.

We believe that its ability to derive long-term from short-term security, also known as everlasting security 14 may be the most attractive aspect of QKE systems from a security perspective.

The baby... The advent of public-key cryptography revolutionized secure telecommunications, by vastly simplifying the problems of key distribution and key management: Alice and Bob no longer needed to pre-share a symmetric key. Instead, Alice could publish her own public key, and that would be sufficient for her to receive encrypted messages from anyone who got a hold of it.

Of course, "publishing" a public key is easier said than done, but publickey cryptography helps solve this problem, too. A signature scheme can be used in conjunction with a network of trusted third parties to help Bob be certain that he has Alice's legitimate public key 15 This is probably the reason Rivest [31] wrote, "The notion of a digital signature may prove to be one of the most fundamental and useful inventions of modern cryptography."

...the bathwater. There is a price to pay for the advantages of a publickey infrastructure. Security necessarily depends on assumptions about the hardness of certain mathematical problems; proofs that such problems are actually hard seem to be beyond the reach of theoretical computer scientists.

\footnotetext{
${ }^{14}$ The term "everlasting security" has been used in the context of the bounded storage model (see, e.g., Ref. 28]), where, e.g., it describes the case where encryption is secure even if the adversary, at some later time, learns the pre-shared symmetric key, as long as, at the time of transmission of the ciphertext, the adversary has bounded storage capability (see Ref. 29]). The term seems equally well suited to QKE.

${ }^{15} \mathrm{On}$ the Internet, this works as follows. Bob's web-browser comes from the manufacturer pre-loaded with the public key of a trusted third party Charlie. When Bob wants to communicate with Alice, she shows Bob a certificate which contains her purported public key and Charlie's signature of the certificate, which also contains Alice's name (and other uniquely identifying and publicly-agreed-upon details about Alice). Bob checks that Alice's public key is valid by verifying Charlie's signature using the pre-loaded public key. In this context, signature schemes are said to offer "manageable persistence" (via digital signature) of the binding of a name and a key [30].
} 
After Peter Shor discovered an efficient quantum algorithm for factoring and computing discrete logarithms in 1994, QKE protocols, the earliest of which dates back to 1984, received renewed interest. Most literature on QKE that appeared in the 1990s and early 2000s focussed on protocols in the class $\mathrm{q}-\mathrm{AKE}_{\mathrm{sym}}$. And rightfully so: it is remarkable that symmetric initial keys can be expanded into much larger, independent, and information-theoretically secure secret keys in band by exploiting quantum mechanics. As such, these articles, through their reference to Shor's discovery, may have been seen as suggesting that all computational assumptions should be jettisoned at the earliest opportunity - for who knew what problems might next succumb to the power of a quantum computer?

A new spin on quantum cryptography. It was known (though perhaps not widely) that insisting on unconditional security was not the only way forward in order to ensure reasonable security against quantum attacks. It was evident that public-key signature schemes could be used to authenticate the classical channel in a QKE protocol, and that such a system would have some attractive features; this idea first appeared in the literature in Ref. [2]. Indeed, in light of Theorem 12 and Table 2, and assuming Conjecture 15 is true, this idea becomes rather more striking:

- Quantum cryptography is the only known way to achieve (quantumresistant) private communication in a public-key infrastructure with the minimal computational assumptions.

(If in addition Conjecture 14is true, then the word "known" can be dropped.) In other words, with some abuse of the metaphor, quantum cryptography potentially allows us to throw out some of the bathwater-i.e., primitives with a trapdoor property - while keeping most of the baby -i.e., authenticated encryption without symmetric initial keys - and no classical scheme is known to accomplish this. At the very least, quantum cryptography certainly allows us to sidestep the question of the necessity of trapdoor predicates for secret key agreement (or trapdoor functions for trapdoor predicates [32]). We view this as strengthening the case for signed QKE.

If public-key encryption exists... If trapdoor predicates do exist and are secure in the long term, we note that Advantages 1 through 4 can variously be achieved by sc-AKE-protocols to at least some degree. However, in this case, QKE protocols may have other advantages over classical ones. Because the secret key $s$ generated in a q-AKE-protocol is independent of the 
classical communication $c$, there is no mathematical way to connect these two quantities or - attribute - the secret key to Alice's and Bob's publicly readable discussion; we say that the secret key is nonattributable 16

There are two ways in which a secret key may be considered attributable: it is attributable to Alice's and Bob's public discussion (through its dependence on the classical communication) and it is attributable to Alice and/or Bob (because they participated in the classical communication). For the former way, we just use the term attributable to describe the secret key; for the latter way, we say the secret key is party-attributable. If the classical communication is authenticated via a signature scheme, then the secret key may be party-attributable in a provable way, or provably party-attributable. If the secret key is subsequently used in an encryption scheme to encrypt a plaintext, then we say that the plaintext is (party- or provably party-) attributable whenever the secret key is.

Because q-AKE-protocols do not produce an attributable secret key, a q-

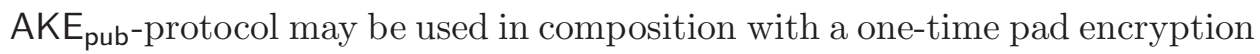
scheme, and then the secret key (and hence the plaintext) would never be attributable. No totally classical scheme can achieve the same thing, i.e., non-party-attributable, public-key, secure communication.

For symmetric-key ciphers where the bit-length of the secret key is much smaller than the bit-length the message (e.g., AES), the cipher itself provides a subroutine for recognizing the secret key (i.e., if a candidate secret key $s^{\prime}$ decrypts the ciphertext to something sensible, then with high probability $s^{\prime}$ equals the actual secret key). If the secret key was produced by a sc-AKE $\mathrm{pub}$ protocol, then the secret key (and hence the plaintext) are provably partyattributable given the secret key; however, if the secret key was produced by a $\mathrm{q}-\mathrm{AKE}_{\text {pub-protocol, }}$ it is not attributable at all. This is a potential advantage of using QKE to generate AES keys.

\footnotetext{
${ }^{16}$ In Ref. 33, Beaver discusses "deniability" (see Refs 34, 35]) of QKE, which is similar to nonattributability. However, in that paper, it is assumed that Alice and Bob keep a record of their qubit-measurement outcomes (often called "raw key bits") made during the protocol and that, if Alice and Bob are to deny that a particular secret key was established, this record must be consistent with any measurements made by an eavesdropper, i.e., someone who is forcing Alice or Bob to reveal the secret key (or the plaintext encrypted by it). We assume that Alice and Bob do not keep such records and that it is sufficient that the forcer cannot provide evidence that attributes a particular secret key to the classical communication; any measurement on the quantum channel that the forcer made is not publicly verifiable, so we do not view its outcome as part of the public record. In other words, in our model, Alice and Bob need not provide evidence to support their (tacit) denial. Incidentally, Beaver concludes that the standard QKE protocols do not provide deniability in his model.
} 
Closing Remarks. Recall the objections to QKE that we listed earlier (see Page 2). We have addressed Objection 4 early on, by highlighting the fundamental distinction between in-band and out-of-band key establishment protocols. We believe there exist (or will exist) applications where in-band generation of entropy is desirable.

Objections 2 and 3 both propose using (potentially very long) symmetric initial keys in OOB or PGE protocols. We have presented a considerable list of advantages that QKE has over these protocols.

Objection 1 is the strongest one, but it relies on the computational assumption of a trapdoor predicate, which (until any lower bounds are proven) incurs risk when public-key encryption is used for long-term secrets. The field of quantum algorithms is still relatively young, so it is probably unwise to assume any particular candidate trapdoor predicate with a particular set of parameters is secure (the recent discovery of a subexponential-time quantum algorithm for elliptic curve isogenies supports this perspective [36]). However, in addition to these standard counter-arguments for Objection 1, we have shown that QKE may offer the benefit of nonattributability in scenarios where no purely classical scheme can. We also note that it is conceivable that, in the future, a q-AKE-system may be more efficient (i.e. have a higher secret key rate) than a sc-AKE-system, as public-key encryption is known to be rather slow. As well, q-AKE-systems may be more costeffectively resistant to side-channel attacks, which are notoriously difficult to defend against in the classical world.

The debate on the merits of QKE may have suffered from a focus on unconditional security, which may have given the impression that it is of no value to practical cryptography. The message from classical cryptographers has been loud and clear: the pre-sharing of symmetric keys is costly and thus to be avoided in the majority of key-establishment applications: e.g., Paterson et al. [2] wrote, "[Quantum key establishment], when unconditionally secure, does not solve the problem of key distribution. Rather, it exacerbates it, by making the pre-establishment of symmetric keys a requirement." They also wrote, "It is likely that using [QKE] with public key authentication [...] has security benefits [...]. However, [QKE] loses much of its appeal in [this setting], as the overall system security is no longer guaranteed by the laws of quantum physics alone." Our article is completely in accordance with the former comment and, with regard to the latter comment, expands on the "benefits" of signed QKE in order to bolster its "appeal". As such, we hope to have firmed up the middle ground between unconditionally-secure QKE and computationally-secure classical key establishment in the "quantum debate". 


\section{References}

[1] Charles H. Bennett and Peter W. Shor. Privacy in a quantum world. Science, 284(5415):747-748, 1999.

[2] K. G. Paterson, F. Piper, and R. Schack. Quantum cryptography: a practical information security perspective. In M. Zukowski, S. Kilin, and J. Kowalik, editors, Quantum Communication and Security, 2007.

[3] Romain Alleaume, Jan Bouda, Cyril Branciard, Thierry Debuisschert, Mehrdad Dianati, Nicolas Gisin, Mark Godfrey, Philippe Grangier, Thomas Länger, Anthony Leverrier, Norbert Lëkenhaus, Philippe Painchault, Momtchil Peev, Andreas Poppe, Thomas Pornin, John Rarity, Renato Renner, Gregoire Ribordy, Michel Riguidel, Louis Salvail, Andrew Shields, Harald Weinfurter, and Anton Zeilinger. Secoqc white paper on quantum key distribution and cryptography. arXiv:quant-ph/0701168, 2007.

[4] D. Stebila, M. Mosca, and N. Lutkenhaus. The case for quantum key distribution. In M. Zukowski, S. Kilin, and J. Kowalik, editors, Proceedings of QuantumComm 2009 Workshop on Quantum and Classical Information Security, volume 36, 2009.

[5] D. Bernstein. Cost-benefit analysis of quantum cryptography. In Workshop on Classical and Quantum Information Assurance Foundations and Practice, Schloss Dagstuhl, July 2009. http://www.dagstuhl.de/Materials/index.en.phtml?09311.

[6] N. Lütkenhaus and A. J. Shields. Focus on quantum cryptography: Theory and practice. New Journal of Physics, 11(4):045005.

[7] O. Goldreich. Foundations of cryptography (Volume I): Basic tools. Cambridge University Press, Cambridge, 2001.

[8] O. Goldreich. Foundations of cryptography (Volume II): Basic applications. Cambridge University Press, Cambridge, 2004.

[9] Mihir Bellare and Phillip Rogaway. Entity authentication and key distribution. In CRYPTO '93: Proceedings of the 13th annual international cryptology conference on Advances in cryptology, pages 232-249, New York, NY, USA, 1994. Springer-Verlag New York, Inc. 
[10] Ran Canetti and Hugo Krawczyk. Analysis of key-exchange protocols and their use for building secure channels. Cryptology ePrint Archive, Report 2001/040, 2001. http://eprint.iacr.org/2001/040.

[11] A. J . Menezes, P. van Oorschot, and S. Vanstone. Handbook of Applied Cryptography. CRC Press LLC, Boca Raton, 1996.

[12] Russell Impagliazzo and Steven Rudich. Limits on the provable consequences of one-way permutations. In CRYPTO '88: Proceedings of the 8th Annual International Cryptology Conference on Advances in Cryptology, 1990.

[13] C. H. Bennett and G. Brassard. Quantum cryptography: Public key distribution and coin tossing. In Proceedings of IEEE International Conference on Computers, Systems and Signal Processing, pages 175179, New York, December 1984. IEEE Press.

[14] Artur K. Ekert. Quantum cryptography based on Bell's theorem. Phys. Rev. Lett., 67(6):661-663, 1991.

[15] Jörn Müller-Quade and Renato Renner. Composability in quantum cryptography. New Journal of Physics, 11(8):085006.

[16] Ran Canetti. Universally composable security: A new paradigm for cryptographic protocols. Cryptology ePrint Archive, Report 2000/067, 2000. http://eprint.iacr.org/

[17] M. Nielsen and I. Chuang. Quantum Computation and Quantum Information. Cambridge University Press, Cambridge, 2000.

[18] Douglas R. Stinson. Cryptography: Theory and Practice. CRC Press LLC, Boca Raton, 1995.

[19] Brian LaMacchia and Kristin Lauter Anton Mityagin. Stronger security of authenticated key exchange. In ProvSec'0\%: Proceedings of the 1st international conference on Provable security, 2007.

[20] T. Okamoto, K. Tanaka, and S. Uchiyama. Quantum public-key cryptosystems. In Proc. of CRYPTO 2000, volume 1880 of Lecture Notes in Computer Science, pages 147-165. Springer-Verlag, 2000.

[21] S. Goldwasser and S. Micali. Probabilistic encryption. Journal of Computer and Systems Sciences, 28(2):270-299, April 1984. 
[22] Mark N. Wegman and J. Lawrence Carter. New hash functions and their use in authentication and set equality. pages 265-279, 1981.

[23] M. Naor and M. Yung. Universal one-way hash functions and their cryptographic applications. In Proceedings of the 21st Annual ACM Symposium on Theory of Computing, 1989.

[24] J. Rompel. One-way functions are necessary and sufficient for secure signatures. In STOC '90: Proceedings of the twenty-second annual ACM symposium on Theory of computing, 1990.

[25] Renato Renner. Security of quantum key distribution. PhD thesis, Swiss Federal Institute of Technology, 2005.

[26] Daniel J. Bernstein, Johannes Buchmann, and Erik Dahmen. Post Quantum Cryptography. 2008.

[27] O. Goldreich, S. Micali, and A. Wigderson. Proofs that yield nothing but their validity or all languages in np have zeroknowledge proofs. Journal of the ACM, 1991.

[28] Christian Cachin and Ueli M. Maurer. Unconditional security against memory-bounded adversaries. In CRYPTO '97: Proceedings of the 17th Annual International Cryptology Conference on Advances in Cryptology, 1997.

[29] Yan Zong Ding and Michael O. Rabin. Hyper-encryption and everlasting security. In STACS '02: Proceedings of the 19th Annual Symposium on Theoretical Aspects of Computer Science, pages 1-26, London, UK, 2002. Springer-Verlag.

[30] Carlisle Adams and Steve Lloyd.

[31] Ronald L. Rivest. Handbook of Theoretical Computer Science, chapter Cryptography, pages 717-755. Elsevier, 1990.

[32] Y. Gertner, T. Malkin, and O. Reingold. On the impossibility of basing trapdoor functions on trapdoor predicates. In IEEE Press, editor, Proc. 42nd Annual IEEE Symposium on the Foundations of Computer Science (FOCS '01), pages 126-135, 2001.

[33] Donald Beaver. On deniability in quantum key exchange. In Proceedings of the International Conference on the Theory and Applications of Cryptographic Techniques: Advances in Cryptology, EUROCRYPT '02, 2002. 
[34] Ran Canetti, Cynthia Dwork, Moni Naor, and Rafail Ostrovsky. Deniable encryption. In In Crypto 97, pages 90-104. Springer-Verlag, 1996.

[35] Marek Klonowski, , Przemyslaw Kubiak, and Miroslaw Kutylowski. Practical deniable encryption. In Viliam Geffert, Juhani Karhumki, Alberto Bertoni, Bart Preneel, Pavol Nvrat, and Mria Bielikov, editors, SOFSEM 2008: Theory and Practice of Computer Science, Lecture Notes in Computer Science. Springer Berlin / Heidelberg.

[36] Andrew Childs, David Jao, and Vladimir Soukharev. Constructing elliptic curve isogenies in quantum subexponential time. in preparation, 2010. 